\title{
Acknowledgement to the Reviewers
}

The editors gratefully acknowledge the valuable contributions of the following reviewers to the evaluation of the papers submitted to the Journal of Nutrigenetics and Nutrigenomics.

A. Leikin-Frenkel, Tel Aviv, Israel
C.M. Aguilera, Granada, Spain
C.L. Amaral, São Paulo, Brazil
F. Bolaños-Jiménez, Nantes, France
L. Broersen, Utrecht, Netherlands
M.S. Choi, Daegu, Republic of Korea
C. Cominetti, Goiânia, Brazil
R. Curi, São Paulo, Brazil
R. De Caterina, Chieti, Italy
A. El-Sohemy, Toronto, Ont., Canada
L. Ferguson, Auckland, New Zealand
N.L. Garneau, Denver, Colo., USA
W. Garver, Albuquerque, N.Mex., USA
U. Genick, Zurich, Switzerland
X. Han, Peking, China
H.H. Hermsdorff, Viçosa, Brazil
T. Huang, Boston, Mass., USA
N. Hudson, St. Lucia, Qld., Australia
X. Jiang, Brooklyn, N.Y., USA
T. Kühn, Heidelberg, Germany
C.-Q. Lai, Boston, Mass., USA
P. Lin, St. Louis, Mo., USA
Y. Liu, Shanghai, China
J. Lovegrove, Reading, UK
D. Ma, Guelph, Ont., Canada
E.C.M. Mariman, Maastricht, Netherlands
F. Marotta, Milan, Italy

J.A. Martinez, Pamplona, Spain

C. Meplan, Newcastle upon Tyne, UK

L. Migliore, Pisa, Italy

C. Nonino, Ribeirão Preto, Brazil

N. Osumi, Sendai, Japan

L. Perusse, Quebec, Que., Canada

P. Prieto-Hontoria, Pamplona, Spain

M.P. Rayman, Surrey, UK

G. Rimbach, Kiel, Germany

I. Rudkowska, Quebec, Que., Canada

F.J. Sanchez-Muniz, Madrid, Spain

W.H.M. Saris, Maastricht, Netherlands

E. Solis, Monterrey, Mexico

B. Stefanska, West Lafayette, Ind., USA

N. Turner, College Station, Tex., USA

M. Uusitupa, Kuopio, Finland

I. Valladolid-Acebes, Stockholm, Sweden

B. Vizmanos, Guadalajara, Mexico

M.-C. Vohl, Quebec, Que., Canada

J.-B. Wan, Taipa, Macau

E. Wu, Fargo, N.Dak., USA

A. Xu, Hong Kong, China

J. Yang, Yinchuan City, China

A.M. Zivkovic, Davis, Calif., USA 